\title{
INTENSITY AND BUNCH-SHAPE DEPENDENT BEAM LOSS SIMULATION FOR THE SIS100
}

\author{
G. Franchetti*, I. Hofmann, A. Orzhekhovskaya, P. Spiller, GSI, Darmstadt, Germany
}

\section{Abstract}

We have studied the combined influence of magnet nonlinearities, space charge and bunch shapes consistent with different RF scenarios on the long-term loss in the planned SIS100 synchrotron of the FAIR project. The simulation is a $3 \mathrm{D}$ tracking with "frozen-in" space charge calculation employing the MICROMAP code. Comparing a oneharmonic RF scenario with an alternative double-harmonic scenario we find that for the same absolute beam loss roughly twice the number of particles can be stored in the double-RF system. Moreover, a barrier bucket RF scenario is found to be loss-free. This is due to the fact that loss is caused here by space-charge induced periodic resonance crossing, which is absent for the strictly flat bunch profile of the barrier case.

\section{THE SIS100 RING}

In the FAIR project [1] the SIS100 synchrotron [2] is composed of six super-periods made by 15 doublet focusing DF structures and 20 dipoles. The sc dipole magnet of Nuklotron type have apertures of $130 \mathrm{~mm}$ x $65 \mathrm{~mm}$. The length of the ring is $11 / 2$ times the SIS18 circumference and the magnetic rigidity $B \rho=100 \mathrm{Tm}$. In Fig. 1 is shown the layout of the existing facility and the SIS100 in the new facility.

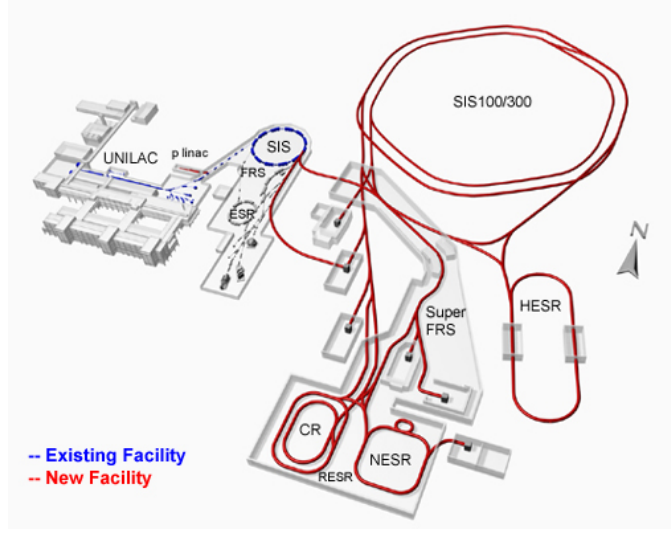

Figure 1: FAIR project and SIS100.

In the research program foreseen in FAIR, SIS100 has to deliver a primary beam of $10^{12}$ ions $\mathrm{U}^{+28}$ per second at energies from 400 to $2700 \mathrm{MeV} / \mathrm{u}$. The technical features of SIS100 require vacuum quality in the XHV range with a base pressure of $P=10^{-12}$ mbar. The large ionization cross section of $\mathrm{U}^{+28}$ makes the interaction of the ion beam

\footnotetext{
*g.franchetti@gsi.de
}

with residual gas atoms critical. Experience with the SIS18 has shown that during high intensity operation ion desorption processes leads to significant vacuum degradation and limitation of beam lifetime. Beam loss control therefore becomes a delicate issue. In the present scenario the RF has $h=10$. After 4 individual transfers from the SIS 18 to SIS100, 8 buckets will be filled and 2 will remain empty. The nominal intensity is $\sim 1.25 \times 10^{11}$ ions and transverse emittances of $\epsilon_{x / y}=50 / 20 \mathrm{~mm}$-mrad. This requires some optimization of the RF to keep the transverse tuneshifts in the still acceptable range of $\Delta Q_{y}=-0.2,-0.4$. With 1 second storage time in the SIS100 space charge sets unprecedented challenges to beam dynamics and lattice design. In order to explore these issues a campaign of experiments was started in 2002 at the CERN-Proton Synchrotron (PS) in a CERN-GSI collaborative effort which could be used for benchmarking our theoretical model versus an experiment with enforced beam loss. By exciting an external 4th order resonance it was shown that a bunched beam of emittances $\epsilon_{x / y}=25 / 10 \mathrm{~mm}$-mrad (normalized at $2 \sigma$ ) and a tuneshift $\Delta Q_{y}=0.075$ reduces its intensity by $32 \%$ during 1 second storage when the bare tunes are $Q_{x 0}=6.265, Q_{y 0}=6.12$ [3]. The beam loss was attributed to the combined effect of beam space charge and longitudinal motion, which creates a periodic migration of islands in the transverse phase space. Particles trapped into islands are brought to the dynamic aperture and eventually lost [4]. The basic ingredient in this loss mechanism is the longitudinal motion, which pushes particles through bunch regions with different ion density. Consequently, beam loss is expected to be affected by the longitudinal bunch density profile. We consider here three scenarios: 1) Single RF harmonic system (1-RF); 2) Double RF harmonic system (2-RF); 3) Barrier bucket (B-B).

\section{SIS100 NONLINEAR LATTICE AND WORKING POINT}

The SIS100 nonlinearities are modeled as follows: in the sc bends nonlinear kicks are set in the center of each magnet. The multipoles are computed for $10 \%$ of the magnet excitation. The order of the expansion is taken up to 13th order. Skew components are at the moment not included. The effect of the sagitta is modeled by a coordinate shift of $8 \mathrm{~mm}$, which brings the particle into outer regions of the bend sampling stronger nonlinear components. Quadrupole fringe fields are modeled as well, but skew components are ignored [5]. The choice of the working point is chosen such to avoid systematic resonances. Design constrains for the present lattice allow the SIS100 tunes to be taken in the quadrant $26<Q_{x 0}<27,17<Q_{y 0}<18$. 
The acceptance is $179 / 47 \mathrm{~mm}-\mathrm{mrad}$. A detection of all the resonances excited is obtained by a scan of the dynamic aperture (DA) in this working region. As imperfections have to be added to the systematic strength of the nonlinear components in real magnets, we have perturbed the $b_{n}$ of each magnet with an error belonging to a Gaussian distribution with a variance of $10 \%$ and zero average [5]. We then obtain a possible configuration of the SIS100 nonlinear lattice made by systematic+random nonlinear errors and use this lattice throughout this study. We compute the DA for on-momentum particles in $10^{4}$ turns. The DA is defined as the radius (in normalized coordinates) of the largest circle inscribed inside the domain of stable initial conditions in $N$ turns [6]. We express the DA in terms of the beam $\sigma$, for equal emittances $\epsilon_{x / y, r m s}=8.75 \mathrm{~mm}$-mrad [2]. The results of the DA scan is shown in Fig. 2. The DA is horizontally at the level of the beam pipe (red area). Only the fifth order systematic resonance $Q_{x}+4 Q_{y}=96$ is strongly excited reducing the DA to the level of the beam edge. As understood in our previous study [4] the most dangerous effects induced by space charge happen when the bare tune is just above a resonance line. We therefore avoid setting the working point above the line $Q_{x}+4 Q_{y}=96$ as this resonance is likely to produce beam loss even without the contribution of space charge. However it may be assumed that weak high order resonance as the $3 Q_{x}+6 Q_{y}=186$ is much less dangerous, hence we choose the working point $Q_{x 0}=26.45, Q_{y 0}=17.83$ right above this resonance line in order to test the three RF scenarios (see Fig. 2). The incoherent tunespread of $\Delta Q_{x}=0.2$ also crosses the systematic resonance $3 Q_{x}+4 Q_{y}=150$, which appears even weaker in Fig. 2.

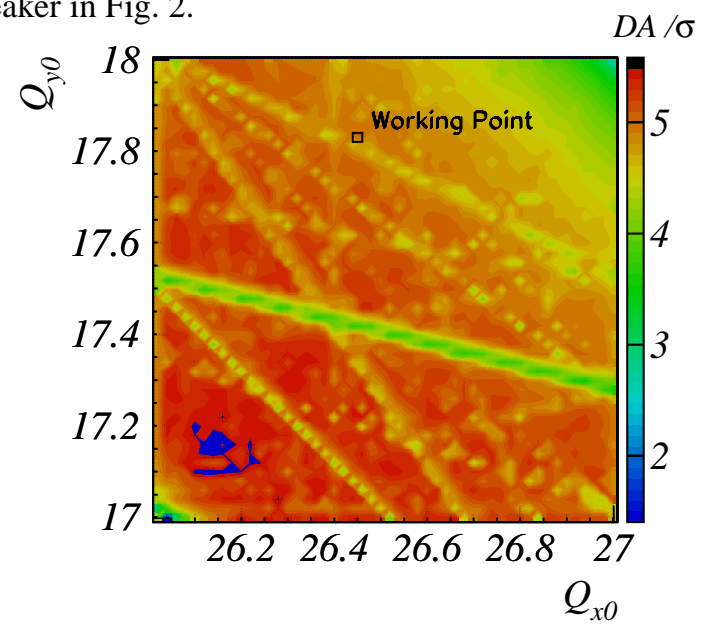

Figure 2: DA of SIS100 versus working point.

\section{BEAM LOSS DURING BUNCH STORAGE FOR DIFFERENT RF SYSTEM}

The total ions stored in the SIS100 is planned to be $10^{12}$ in 1 second, which corresponds to approximately $10^{5}$ turns. We consider a bunch with nominal intensity and emittance and a momentum spread at $1 \sigma$ of $2.5 \times 10^{-4}$. In order to compare the beam loss we use for each RF system a matched bunch distribution with momentum spread of $4 \times 10^{-4}$ and a number of ions consistent with a maximum incoherent tuneshift of $\Delta Q_{y}=-0.2$. In this way the effect of dispersion and chromaticity plays the same role in all three cases. In the single RF system we keep for simplicity the bunch in the linear part of the bucket. In order to obtain the required maximum momentum spread the bunch length must be $158 \mathrm{~m}$ (at $5 \sigma)$. The tuneshift of $\Delta Q_{y}=-0.2$ is reached with $0.44 \times 10^{11}$ ions per bunch. We assume a synchrotron tune of $Q_{s}=10^{-3}$. The double RF system is modeled as in [8] keeping the amplitude of the basic harmonic the same as in the 1-RF system. The longitudinal bunch edge has been chosen at the phase of $\pi / 2$, and the consistent bunch length for the required momentum spread is $249 \mathrm{~m}$. For the same longitudinal peak intensity the 2-RF case has a number of ions 2.19 bigger of that for the $1-\mathrm{RF}$ case. The tuneshift of $\Delta Q_{y}=-0.2$ is now reached with $1.0 \times 10^{11}$ ions per bunch. The barrier bucket is modeled as an ideal reflecting wall in phase space, with the longitudinal matched distribution uniform in $z$ and Gaussian in $\delta p / p$. The bunch length is chosen $190 \mathrm{~m}$ and the spread in $\delta p / p$ is measured as 3 times the variance. The total number of ions per bunch is $1.0 \times 10^{11}$ which is consistent with equal maximum tuneshift of $\Delta Q_{y}=-0.2$. In Table 1 we summarize the parameters of the bunched beam in the three RF systems. In order to prevent an

Table 1: Main characteristics of beam stored in the three RF systems.

\begin{tabular}{lccc}
\hline RF & N. ions $\times 10^{11}$ & Length $[\mathrm{m}]$ & Synch. tune \\
\hline 1-RF & 0.44 & $158(5 \sigma)$ & $10^{-3}$ \\
2-RF & 1.0 & 249 & $10^{-3}$ (main RF) \\
B-B & 1.0 & 190 & - \\
\hline
\end{tabular}

initial beam loss caused by the SIS100 mechanical acceptance the transverse distribution for all the bunches is a truncated Gaussian. We cut the particle distribution in energy at $2.5 \sigma$. A test simulation using this distribution at low intensity showed that no loss is found. The space charge calculations are performed by using a $2.5 \mathrm{D}$ model [7] in which the electric field is calculated with a 2D frozen model of a Gaussian beam with arbitrary axes. For each macro-particle the space charge is created by a local coasting beam of transverse sizes $\sqrt{\beta_{x} \epsilon_{x}}, \sqrt{\beta_{y} \epsilon_{y}}$ and intensity consistent with the bunch local density. We have found that for a bunch with typical aspect ratio $r_{x} / r_{z} \sim 10^{-4}$ and wavelength of $\beta_{x}, \beta_{y}$ as in SIS100 this approximation is satisfactory. With this space charge modeling the effect of lattice induced twisting of the bunch along the longitudinal direction is included. We simulate the beam evolution and estimate the beam loss in the first $2.5 \times 10^{4}$ turns for each of the 3 RF systems above described. Each tracking was performed by using 2000 macro-particles in the MICROMAP code with 86 space charge kicks per lattice period. In Fig. 3 the relative beam loss in $\%$ is given as function of turns. The 1-RF scenario exhibits double relative loss than the 2- 
RF. However, as the number of ions in the 2-RF case is almost twice that of the 1-RF case, the absolute beam loss is almost equal in the two scenarios. Fig. 3 shows also that

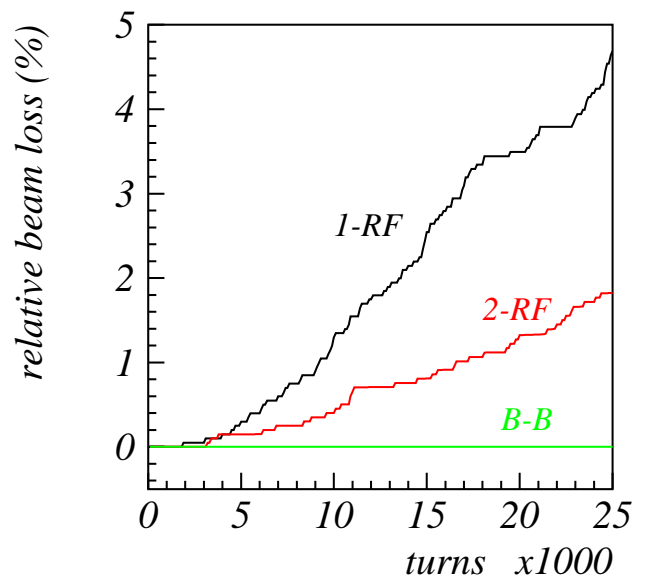

Figure 3: Beam loss at injection as function of turns for different RF systems with the same $\Delta Q_{y}$.

the B-B scenario has no beam loss. This finding is consistent with the lack of resonance crossing as single particle tunes jump instantaneously at the bunch ends due to chromaticity and reflecting walls in the phase space.

As the simulations have been performed only up to $2.5 \times 10^{4}$ turns, it is useful to estimate the total amount of particles which can be lost if the bunch is stored for unlimited time. This may happen for all particles which periodically cross the resonance $3 Q_{x}+6 Q_{y}=186$ [4]. The condition therefore is a sufficiently large synchrotron amplitude, noting that a particle reaching the bunch end will have $Q_{y}=Q_{y 0}$. To estimate the number of these particles we have used a smooth focusing approximation and calculated space charge depressed tunes as function of amplitudes. This estimate for the asymptotic relative beam loss in the 1-RF scenario gives $35 \%$, and $11 \%$ for the $2-\mathrm{RF}$ scenario; the absolute beam loss is $0.15 \times 10^{11}$, respectively $0.11 \times 10^{11}$. Due to the assumption of equal $\Delta Q_{y}$ the 2-RF scenario allows the storage of twice the number of ions, but our result shows that noetheless the absolute asymptotic beam loss is reduced by $25 \%$. These results have been derived for $\Delta Q_{y}=-0.2$, but the comparison can be easily extended to other $\Delta Q_{y} \neq-0.2$ by rescaling consistently the incoherent tuneshifts. The resulting change of the number of stored particles is taken into account for computing absolute beam loss. In Fig. 4 we summarize the asymptotic relative beam loss for the 1-RF and 2-RF scenarios. We find the existence of a tuneshift threshold $\Delta Q_{y, t h}=-0.12$, which divides the efficiency of the two $\mathrm{RF}$ scenarios (in terms of absolute asymptotic beam loss) into two regimes: for $\left|\Delta Q_{y}\right|<\left|\Delta Q_{y, t h}\right|$ the 1-RF scenario causes lower beam loss than the 2-RF, and vice versa for $\left|\Delta Q_{y}\right|>\left|\Delta Q_{y, t h}\right|$. Note that in both RF systems the asymptotic beam loss drops to zero when $\left|\Delta Q_{y}\right| \leq 0.05$. This means that the full tunespread is above the resonance and no periodic crossing is possible for any particle. For

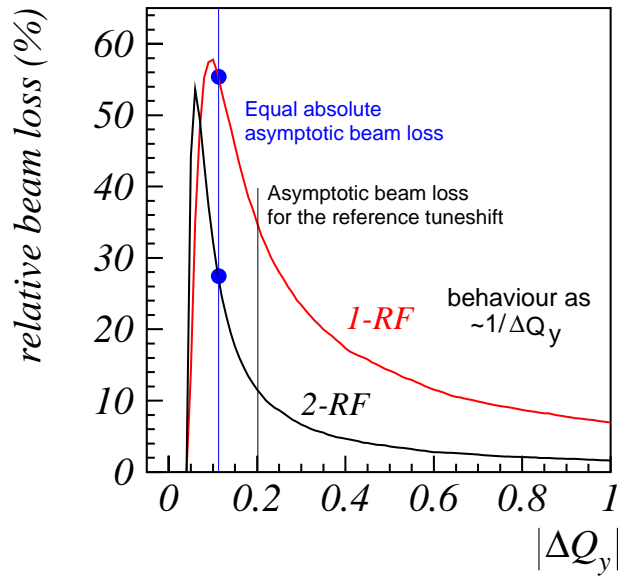

Figure 4: Asymptotic beam loss limit for 1-RF and 2-RF systems.

tunespreads larger then $\left|\Delta Q_{y}\right|>0.3$ the asymptotic absolute beam loss in the two scenarios becomes constant: $0.16 \times 10^{11}$ for $1-\mathrm{RF}$ and $0.80 \times 10^{11}$ for the 2 -RF. Consequently the relative beam loss scales as $1 /\left|\Delta Q_{y}\right|$.

\section{CONCLUSION}

From this study we conclude that the B-B system is the most attractive as it produces no beam loss. The trade-off between 1-RF and 2-RF systems may not become effective for large $\Delta Q_{y}$ if several resonance lines overlap with the tunespread. In addition, with beam loss of tens of percent the assumption of a space charge frozen model becomes questionable and an update of beam parameters during the simulation may be necessary, which is the subject of future studies.

We are thankful to A. Luccio (AC) and N. D'Imperio (CDIC), BNL for the use of the Galaxy computer system.

\section{REFERENCES}

[1] P. Spiller, these Proceedings.

[2] SIS100 Technical Report, to be published.

[3] G. Franchetti et al., Phys. Rev. ST Accel. Beams 6, 124201 (2003).

[4] G. Franchetti et al., to be published in Proc. of 33rd ICFA workshop, 2004.

[5] G. Franchetti, and I. Hofmann, Proc. of EPAC 2004, p. 1978, (2004).

[6] J. P. Koutchouk, Proc. of PAC 99, p. 2, (1999); M. Giovannozzi, W. Scandale, and E. Todesco, Phys. Rev., E57, 3432 3443 (1998).

[7] A. Orzhekhovskaya, G. Franchetti, Proc. of EPAC 2004, p. 1975, (2004).

[8] S.Y.Lee, Accelerator Physics, World Scientific, p. 298 (1998). 